\title{
TESTE DE DETERIORAÇÃO CONTROLADA NA DETERMINAÇÃO DO VIGOR EM SEMENTES DE ALGODÃO ${ }^{1}$
}

\author{
ALEK SANDRO DUTRA², SEBASTIÃO MEDEIROS FILHO ${ }^{3}$
}

\begin{abstract}
RESUMO - A avaliação do vigor tem sido ferramenta fundamental dentro de programas de controle de qualidade de sementes, e o teste de deterioração controlada pode ser utilizado neste processo. A presente pesquisa foi desenvolvida com o objetivo de verificar a eficiência do teste de deterioração controlada para a avaliação do vigor de sementes de algodão. A qualidade inicial de cinco lotes de sementes foi avaliada pelos testes de germinação, primeira contagem de germinação, emergência em campo, índice de velocidade de emergência e peso da matéria seca de plântulas. Foram ajustados os teores de águas das sementes para 20 e $24 \%$, sendo então submetidas à deterioração controlada em banho-maria, a 40 e $45^{\circ} \mathrm{C}$, por 24 e 48 horas. A combinação de $24 \%$ de teor de água na semente por 48 horas e $40^{\circ} \mathrm{C}$ no teste de deterioração controlada é eficiente para detectar diferenças de vigor entre lotes de sementes de algodão
\end{abstract}

Termos para indexação: Gossypium hirsutum, análise de sementes, potencial fisiológico.

\section{CONTROLLED DETERIORATION TEST IN VIGOR DETERMINATION IN COTTON SEEDS}

\begin{abstract}
Vigor evaluation has been a basic tool in programs for seed quality control, and the controlled deterioration test is an important part of the process. The present research was developed with to verify the efficiency of the controlled deterioration test for the evaluation of cotton seed vigor. The initial quality for five lots of seeds was evaluated by the germination test, germination at first count, emergence in the field, speed of emergence index and seedling dry weight. The seed water content was adjusted tor 20 and $24 \%$, and they were then submitted to controlled deterioration in a water bath at 40 and $45^{\circ} \mathrm{C}$, for 24 and 48 hours. The combination of $24 \%$ of water in the seed for 48 hours and $40^{\circ} \mathrm{C}$ in the controlled deterioration test was efficient for separating the cotton seed lots.
\end{abstract}

Index terms: Gossypium hirsutum, seed analysis, physiological potential.

\section{INTRODUÇÃO}

Os testes de vigor procuram detectar diferenças significativas no potencial fisiológico de lotes com germinação semelhante, fornecendo informações adicionais às proporcionadas pelo teste de germinação. Paralelamente, espera-se que os resultados permitam distinguir com segurança os lotes de alto dos de baixo vigor e que as

${ }^{1}$ Submetido em 03/05/2007. Aceito para publicação em 13/08/2007.

${ }^{2}$ Eng $^{\circ}$. Agrônomo., Dr., UFC, Depto. de Fitotecnia, Cx.Postal 12.168, 60.356001, Fortaleza,CE, Bolsista DCR/CNPq, e-mail: alekdutra@bol.com.br Autor para correspondência. diferenças detectadas estejam relacionadas ao comportamento das sementes durante o armazenamento e após a semeadura (Marcos Filho, 2005).

A maior limitação do teste de germinação, segundo Hampton \& TeKrony (1995), é sua inabilidade para detectar diferenças de qualidade entre lotes com alta germinação. Por isso, têm sido desenvolvidos testes de vigor com o objetivo de identificar possíveis diferenças no potencial fisiológico 
de lotes que apresentam porcentagem de germinação semelhantes, fornecendo informações complementares às obtidas no teste de germinação, para o controle de qualidade das empresas produtoras de sementes de algodão.

Dentre os testes de vigor disponíveis na atualidade, destaca-se o teste de deterioração controlada, desenvolvido inicialmente para avaliar a qualidade de sementes pequenas (Powell e Matthews, 1981), baseando-se em técnica de envelhecimento similar à do teste de envelhecimento acelerado. Neste teste, as sementes são submetidas a alta temperatura e umidade relativa do ar por período de tempo determinado, condições que favorecem o seu umedecimento. No entanto, a absorção de água com velocidades distintas entre amostras da mesma espécie durante o período de envelhecimento, resulta em diferentes intensidades de deterioração. No teste de deterioração controlada, o grau de umidade das sementes é ajustado para um mesmo nível, em todas as amostras e, somente após esse procedimento, são submetidas a alta temperatura (Matthews, 1980; Hampton e TeKrony, 1995; Krzyzanowski e Vieira, 1999).

$\mathrm{Na}$ condução dos testes que avaliam o vigor das sementes, muitos fatores afetam o comportamento das sementes, entre eles o grau de umidade das sementes e a temperatura de incubação. Segundo Rossetto e Marcos Filho (1995), existe uma relação entre grau de umidade das sementes e a temperatura de instalação do teste, pois sementes devem ter o conteúdo inicial de água elevado sob uma determinada temperatura. Porém, se esta temperatura for maior, podem-se utilizar sementes com menor conteúdo inicial de água. TeKrony (1993), considerou que o grau de umidade das sementes deve ser de 15,5\% (base úmida); caso o conteúdo de água inicial não seja esse, sugere que o mesmo seja atingido através do processo de embebição controlada.

O uso de testes de vigor, justifica-se, como alternativa, para a detecção das diferenças de desempenho entre lotes que apresentam resultados semelhantes no teste de germinação (Carvalho e Nakagawa, 2000). Portanto, a utilização de lotes, com porcentagem de germinação equivalentes entre si, constitui premissa a ser atendida em estudos voltados à verificação da capacidade dos testes de vigor fornecerem dados que, propiciando a diferença qualitativa, permitam ordenação hierárquica dos lotes, baseadas no desempenho fisiológico (Caliari e Silva, 2001). Desta forma, o emprego de vários testes de vigor tem se constituído em alternativa usada e recomendada, uma vez que, rotineiramente, os resultados obtidos são desuniformes entre as avaliações (Marcos Filho, 1998).

O presente trabalho foi realizado com o objetivo de verificar a eficiência do teste de deterioração controlada para a avaliação do vigor de sementes de algodão.

\section{MATERIAL E MÉTODOS}

A pesquisa foi desenvolvida no Laboratório de Análise de Sementes do Departamento de Fitotecnia da Universidade Federal do Ceará-UFC, Fortaleza-CE. Foram utilizados cinco lotes de sementes de algodão herbáceo (Gossypium hirsutum L.), das cultivares BRS 200 Marron (lotes 1 e 2), BRS-Verde (lotes 3 e 4) e CNPA 8H (lote 5), fornecidos pelo Centro Nacional de Pesquisa do Algodão/EMBRAPA, gerência de Barbalha-CE.

As avaliações da qualidade das sementes foram realizadas por meio dos seguintes testes: Determinação do teor de água (base úmida) - foi realizado em estufa a $105 \pm 3^{\circ} \mathrm{C} / 24 \mathrm{~h}$ (Brasil, 1992), utilizando-se quatro subamostras de 50 sementes, para cada tratamento. O teor de água das sementes foi determinado antes e após os períodos em banho-maria; Teste de germinação - foram usadas quatro repetições de 50 sementes, semeadas em rolos de papel toalha, tipo Germitest, umedecidos com água o equivalente a 2,5 vezes o peso do substrato seco e colocado para germinar a $25^{\circ} \mathrm{C}$. As avaliações foram realizadas no quarto e no décimo segundo dia, após a semeadura (Brasil, 1992); Primeira contagem de germinação - conduzido juntamente com o teste de germinação, computando-se a porcentagem de plântulas normais, no quarto dia após a semeadura; Emergência de plântulas em campo - utilizaram-se quatro repetições de 50 sementes, distribuídas em canteiros de $10 \times 1 \mathrm{~m}$, com $20 \mathrm{~cm}$ entre repetições, contendo mistura de terra e areia na proporção de 1:1. As irrigações foram feitas sempre que necessário, visando o fornecimento de água para a germinação das sementes e emergência das plântulas. Determinou-se a porcentagem de emergência de plântulas no décimo segundo dia, após a semeadura. Índice de velocidade de emergência - para a determinação deste índice, foram efetuadas contagens diárias das plântulas emersas a partir da instalação do teste de emergência de plântulas em campo, até décimo segundo dia, após semeadura (Nakagawa, 1999). O índice de velocidade de emergência foi calculado conforme Maguire (1962). Peso da matéria seca - foram utilizadas plântulas oriundas do teste de emergência de plântulas em campo coletadas no décimo segundo dia após a semeadura. As plântulas foram colocadas em estufa, com circulação de ar, a $80^{\circ} \mathrm{C}$ até atingiram peso constante, sendo, posteriormente pesadas em balança com precisão de duas casas decimais. Teste de deterioração controlada - as sementes foram colocadas em caixas plásticas $(11 \times 11 \times 3,5 \mathrm{~cm})$, distribuídas em uma camada única sobre tela metálica suspensa em seu interior. As caixas, tampadas e com $40 \mathrm{~mL}$ de água, foram mantidas em incubadora, a $20^{\circ} \mathrm{C}$. Durante o umedecimento artificial, 
os teores de água das sementes foram monitorados, através de pesagens sucessivas, até os valores desejados de 20 e $24 \%$ pelo método da atmosfera úmida (Rossetto et al., 1995). Após este período, cada amostra foi colocada em recipiente aluminizado, fechada hermeticamente, permanecendo por 24 horas em câmara fria $\left(10^{\circ} \mathrm{C}\right)$ para atingir o equilíbrio higroscópico. Logo após, as sementes foram levadas ao banho-maria, nas temperaturas de 40 e $45^{\circ} \mathrm{C}$, por períodos de 24 e 48 horas. Posteriormente, os recipientes com as sementes foram imersos rapidamente em água fria $\left(20^{\circ} \mathrm{C}\right)$ para reduzir a temperatura. Após o período de deterioração, as sementes foram submetidas ao teste de germinação, em rolos de papel toalha, tipo Germitest, umedecidos com água o equivalente a 2,5 vezes o peso do substrato seco e colocado para germinar a $25^{\circ} \mathrm{C}$. As avaliações das plântulas foram realizadas no quarto dia após a semeadura (Brasil, 1992).

A análise de variância foi realizada separadamente para cada teste em delineamento experimental inteiramente casualizado, com quatro repetições e as médias foram comparadas pelo teste de Tukey a $5 \%$ de probabilidade (Banzatto \& Kronka, 2006). Realizou-se a determinação dos coeficientes de correlação simples entre os resultados dos testes de laboratório e da emergência de plântulas em campo.

\section{RESULTADOS E DISCUSSÃO}

Os resultados obtidos nas avaliações da qualidade inicial dos lotes (Tabela 1) revelaram diferenças entre os lotes no potencial fisiológico. Pelo teste de germinação observouse que os lotes 1, 2, 3 e 4 apresentaram porcentagem de germinação estatisticamente semelhante entre si, e o lote 5 a menor germinação $(74 \%)$. O teste de primeira contagem de germinação classificou os lotes em três níveis de vigor: os lotes 1 e 2 de vigor superior, os lotes 3 e 4 como intermediários e o lote 5 de vigor inferior. Analisando os dados do teste de emergência de plântulas em campo observou-se comportamento semelhante aos verificado no teste de germinação, onde o lote 5 apresentou o menor vigor, sendo que entre os demais lotes não houve diferença. No índice de velocidade de emergência verificou-se que os lotes 1 e 2 apresentaram índices superiores, o lote 5 inferior e os lotes 3 e 4 com valores intermediários. Observou-se nos resultados do peso da matéria seca que o lote 1 apresentou maior vigor não havendo diferença entre os demais lotes.

Os teores de água inicial das sementes antes e após o teste de deterioração controlada estão apresentados na Tabela 2. Observa-se que os teores de água após a realização do teste de deterioração controlada, foram praticamente os mesmos do início do teste, ou seja, antes das sementes serem colocadas no banho maria. Isto permite observar que não houve ganho de água entre as sementes, resultando em nível de envelhecimento similar, durante o mesmo período de tempo. Segundo Krzyzanowski e Vieira (1999), o teste de deterioração controlada incorpora melhor o controle do teor de água das sementes e da temperatura durante o envelhecimento quando comparado com o teste de envelhecimento acelerado.

No teste de deterioração controlada por 24 horas a 40 e $45^{\circ} \mathrm{C}$ (Tabela 3 ), observou-se que o lote 5 foi significativamente inferior aos demais lotes, apesar deste, lote 5 , não ter diferido estatisticamente dos lotes 1 e 4, com o uso da temperatura de $40^{\circ} \mathrm{C}$. Desta forma, de modo geral, o período de 24 horas possibilitou classificar os lotes em dois níveis de vigor, ou seja, os lotes 1, 2, 3 e 4 como os de melhor qualidade e o lote 5 como inferior, independentemente da temperatura usada no banho maria. A combinação $24 \mathrm{~h} / 45^{\circ} \mathrm{C}$ tem sido uma das mais utilizadas (Powell e Matthews, 1981), sendo recomendada para sementes de ervilha (Krzyzanowski e Vieira, 1999). Torres (2002), avaliando diferentes condições de deterioração controlada em sementes de melão, verificou que a combinação

TABELA 1. Valores médios do teste de germinação (TG), primeira contagem (PC), emergência em campo (EC) índice de velocidade de emergência (IVE) e peso da matéria seca (PMS), obtidos para cinco lotes de sementes de algodão ${ }^{1}$.

\begin{tabular}{|c|c|c|c|c|c|}
\hline \multirow[t]{2}{*}{ Lotes } & TG & $\mathrm{PC}$ & $\mathrm{EC}$ & IVE & \multirow{2}{*}{$\begin{array}{c}\text { PMS } \\
(\mathrm{g})\end{array}$} \\
\hline & \multicolumn{4}{|c|}{ 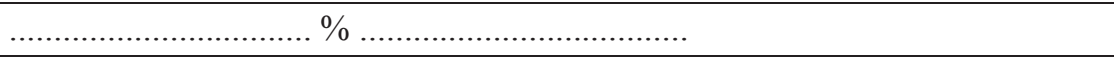 } & \\
\hline 4 & $85 \mathrm{ab}$ & $62 \mathrm{bc}$ & $83 \mathrm{ab}$ & $9,8 \mathrm{bc}$ & $3,48 \mathrm{~b}$ \\
\hline 3 & $84 \mathrm{ab}$ & $75 \mathrm{~b}$ & $85 \mathrm{ab}$ & $10,2 \mathrm{ab}$ & $3,94 \mathrm{~b}$ \\
\hline 5 & $74 \mathrm{~b}$ & $45 \mathrm{c}$ & $74 \mathrm{~b}$ & $8,4 \mathrm{c}$ & $3,74 \mathrm{~b}$ \\
\hline 2 & $97 \mathrm{a}$ & $86 \mathrm{a}$ & $97 \mathrm{a}$ & $11,7 \mathrm{a}$ & $3,84 \mathrm{~b}$ \\
\hline 1 & $95 \mathrm{a}$ & $91 \mathrm{a}$ & $96 \mathrm{a}$ & $11,7 \mathrm{a}$ & $4,82 \mathrm{a}$ \\
\hline $\mathrm{CV}(\%)$ & 7,18 & 11,20 & 6,20 & 7,56 & 8,11 \\
\hline
\end{tabular}

${ }^{1}$ Médias seguidas pela mesma letra nas colunas, não diferem significativamente entre si pelo testes de Tukey ao nível de 5\% de probabilidade de erro. 
TABELA 2. Teor de água médio das sementes, antes e após a realização do teste de deterioração controlada, obtidos para cinco lotes de sementes de algodão.

\begin{tabular}{ccccccc}
\hline & & \multicolumn{3}{c}{ Deterioração controlada } \\
\cline { 3 - 4 } \cline { 5 - 6 } Lotes & Teor de água & \multicolumn{2}{c}{$20 \%$} & & \multicolumn{2}{c}{$24 \%$} \\
\cline { 3 - 4 } \cline { 5 - 6 } & inicial & TAI & TAF & & TAI & TAF \\
\hline 4 & 10,5 & 20,6 & 20,6 & & 24,1 & 24,3 \\
3 & 10,4 & 20,0 & 20,1 & & 24,2 & 24,4 \\
5 & 10,5 & 20,0 & 20,0 & & 24,4 & 24,4 \\
2 & 10,4 & 20,6 & 20,3 & & 24,0 & 24,1 \\
1 & 10,4 & 20,0 & 20,2 & & 24,2 & 24,1 \\
\hline
\end{tabular}

TAI - Teor de água das sementes antes da realização do teste; TAF - Teor de água das sementes após da realização do teste

TABELA 3. Resultados médios do teste de teste de deterioração controlada, após 24 e 48 horas, para os cinco lotes de sementes de algodão com teores de água de $20 \%$ e $24 \%$ e temperaturas de 40 e $45^{\circ} \mathrm{C}$.

\begin{tabular}{|c|c|c|c|c|}
\hline \multirow{3}{*}{ Lotes } & \multicolumn{2}{|c|}{$24 \mathrm{~h}$} & \multicolumn{2}{|c|}{$48 \mathrm{~h}$} \\
\hline & $40^{\circ} \mathrm{C}$ & $45^{\circ} \mathrm{C}$ & $40^{\circ} \mathrm{C}$ & $45^{\circ} \mathrm{C}$ \\
\hline & \multicolumn{2}{|c|}{$20 \%$} & \multicolumn{2}{|c|}{$24 \%$} \\
\hline 4 & $77 \mathrm{ab}$ & $73 \mathrm{a}$ & $84 \mathrm{a}$ & $13 \mathrm{~b}$ \\
\hline 3 & $86 a$ & 89 a & $77 \mathrm{ab}$ & $73 \mathrm{a}$ \\
\hline 5 & $65 \mathrm{~b}$ & $45 \mathrm{~b}$ & $60 \mathrm{~b}$ & $02 \mathrm{c}$ \\
\hline 2 & $83 a$ & $81 \mathrm{a}$ & $88 \mathrm{a}$ & $11 \mathrm{~b}$ \\
\hline 1 & $73 \mathrm{ab}$ & $90 \mathrm{a}$ & $79 \mathrm{a}$ & $05 \mathrm{c}$ \\
\hline CV (\%) & 8,83 & 10,5 & 10,5 & 18,0 \\
\hline
\end{tabular}

Médias seguidas pela mesma letra nas colunas, não diferem significativamente entre si pelo testes de Tukey ao nível de 5\% de probabilidade de erro.

de $45^{\circ} \mathrm{C}$ e período de 24 horas mostrou sensibilidade suficiente para a avaliação do potencial fisiológico das sementes.

Os resultados de germinação de lotes de sementes de algodão, com teor de água de $24 \%$, submetidas à deterioração controlada por 48 horas a 40 e $45^{\circ} \mathrm{C}$ estão exibidos na Tabela 3. Quando o período de deterioração foi aumentado para 48 horas, verificou-se comportamento semelhante em relação ao período de 24 horas, para os lotes 1, 2, 3 e 4 sob temperatura de $40^{\circ} \mathrm{C}$. Neste tratamento, o lote 5 foi o que apresentou a menor porcentagem de germinação $(60 \%)$, muito embora não ter diferido do lote $3(77 \%)$. A germinação das sementes de algodão no tratamento $\left(48 \mathrm{~h} / 45^{\circ} \mathrm{C}\right)$, foi menor que nos demais tratamentos devido ao excessivo período de deterioração na referida temperatura. Quando sementes com $24 \%$ de água foram submetidas à deterioração controlada por 48 horas e $45^{\circ} \mathrm{C}$, o lote 3 foi identificado como o de melhor desempenho seguido pelos lotes 4 e 2 , enquanto que os lotes 3 e 1 tiveram o pior desempenho. Assim, com a deterioração controlada conduzida a $45^{\circ} \mathrm{C}$ por 48 horas, em sementes com teor de água de $24 \%$ foi possível separar os lotes em três níveis de vigor. Segundo
Hampton e TeKrony (1995), lotes de sementes que mantiveram sua germinação após a deterioração são considerados lotes de alto vigor e aqueles que diminuíram a habilidade para germinar são considerados de baixo vigor. Para Santos et al. (2003), o teste de deterioração controlada com grau de umidade inicial das sementes de $20 \%$, período de exposição de 48 horas à temperatura de $45^{\circ} \mathrm{C}$, permite a separação de sementes de feijoeiro com diferentes níveis de vigor.

Pela análise de correlação (Tabela 4), verifica-se que os tratamentos que utilizaram $24 \%$ de teor de água inicial das sementes pelo período de 48 horas de deterioração sob temperatura de $40^{\circ} \mathrm{C}, 20 \%$ de teor de água inicial pelo período de 24 horas e temperaturas de $40^{\circ} \mathrm{C}$ e $45^{\circ} \mathrm{C}$, apresentaram os maiores coeficientes de correlação simples $\mathrm{r}=0,92, \mathrm{r}=$ 0,85 e $\mathrm{r}=0,79$, respectivamente, quando comparado aos resultados do teste de emergência de plântulas em campo. Para Marcos Filho et al. (1984), a correlação significativa indica apenas tendência de variação semelhante entre duas características, de modo que os resultados desta análise não devem ser interpretados isoladamente. 
TABELA 4. Coeficientes de correlação (r) simples entre os tratamentos de deterioração controlada e o teste de emergência de plântulas em campo.

\begin{tabular}{lc}
\hline Tratamentos & $\begin{array}{c}\text { Emergência de plântulas } \\
\text { em campo }\end{array}$ \\
\hline DC $20 \% / 24 \mathrm{~h}-40^{\circ} \mathrm{C}$ & $0,85^{*}$ \\
DC $20 \% / 24 \mathrm{~h}-45^{\circ} \mathrm{C}$ & $0,79^{*}$ \\
DC $24 \% / 48 \mathrm{~h}-40^{\circ} \mathrm{C}$ & $0,92^{* *}$ \\
DC $24 \% / 48 \mathrm{~h}-45^{\circ} \mathrm{C}$ & 0,40 \\
\hline
\end{tabular}

"significativo a $5 \%$ de probabilidade, pelo teste $\mathrm{t} ;{ }^{* *}$ significativo a $1 \%$ de probabilidade, pelo teste $t$.

\section{CONCLUSÃO}

A combinação de $24 \%$ de teor de água na semente por 48 horas e $40^{\circ} \mathrm{C}$ no teste de deterioração controlada é eficiente para detectar diferenças de vigor entre lotes de sementes de algodão.

\section{AGRADECIMENTOS}

Ao CNPq pela bolsa de DCR, a Fundação Cearense de Apoio ao Desenvolvimento Científico e TecnológicoFUNCAP, pelo auxílio à pesquisa e ao Centro Nacional de Pesquisa do Algodão/EMBRAPA na pessoa do Dr. Francisco das Chagas Vidal Neto, pelo fornecimento das sementes.

\section{REFERÊNCIAS}

BANZATTO, D.A.; KRONKA, S.N. Experimentação agrícola. 4. ed. Jaboticabal: FUNEP/UNESP, 2006. 237p

BRASIL. Ministério da Agricultura. Regras para análise de sementes. Brasília: SNAD/DNDV/CLAV, 1992. 365p.

CALIARI, M.F.; SILVA, W.R. Interpretação de dados de testes de vigor na avaliação da qualidade fisiológica de sementes de milho. Revista Brasileira de Sementes, Londrina, v.23, n.1, p.239-251, 2001.

CARVALHO, N.M.; NAKAGAWA, J. Sementes: ciência, tecnologia e produção. Jaboticabal: FUNEP, 2000. 588p.

HAMPTON, J.G.; TEKRONY, D.N. Controlled deterioration test. In: Handbook of vigour tests methods. Zurich: ISTA, 1995. p.70-78.
KRZYZANOWSKI, F.C.; VIEIRA, R.D. Deterioração controlada. In: KRZYZANOWSKI, F.C.; VIEIRA, R.D; FRANÇA-NETO, J.B. (Eds.). Vigor de sementes: conceitos e testes. Londrina: ABRATES, 1999. p.1.1-1.21

MAGUIRE, J.D. Speed of germination-aid selection and evaluation for seedling emergence and vigor. Crop Science, Madison, v.2, n. 1, p.176-177, 1962.

MARCOS FILHO, J. Fisiologia de sementes de plantas cultivadas. Piracicaba: FEALQ, 2005. 495p.

MARCOS FILHO, J. O valor dos testes de vigor. Seed News. Pelotas, n.6, p.32, jul/ago., 1998.

MARCOS FILHO, J. PERCARIN, H.M.C.; KOMATSU, Y.H.; DEMÉTRIO, C.G.B.; FANCELLI, A.L. Testes para avaliação do vigor de sementes de soja e sua relação com a emergência das plântulas em campo. Pesquisa Agropecuária Brasileira, Brasília, v.19, n.5, p.605-613, 1984.

MATTHEWS, S. Controlled deterioration: A new vigour test for crop seeds. In: HABBLETHWAITE, P.D. Seed Production. London: Butterworths. 1980. p.647-660.

NAKAGAWA, J. Testes de vigor baseados no desempenho das plântulas. In: KRZYZANOWSKI, F.C.; VIEIRA, R.D.; FRANÇA NETO, J.B. (Eds.). Vigor de sementes: conceitos e testes. Londrina: ABRATES, 1999. p.2.1 - 2.24.

POWELL, A.A.; MATTHEWS, S. Evaluation of controlled deterioration a new vigour test for small seeds vegetables. Seed Science and Technology, Zurich, v.9, n.2, p.633-640, 1981.

TORRES, S.B. Métodos para avaliação do potencial fisiológico de sementes de melão. Piracicaba, 2002. 103f. Tese (Doutorado em Agronomia), Escola Superior de Agricultura “Luiz de Queiroz”, Universidade de São Paulo, 2002.

ROSSETTO, C.A.V.; FERNANDES, E.M.; MARCOS FILHO, J. Metodologias de ajuste do grau de umidade e comportamento das sementes de soja no teste de germinação. Revista Brasileira de Sementes, Brasília, v.17, n.2, p.171-178, 1995.

ROSSETTO, C.A.V.; MARCOSFILHO, J.Comparação entre os métodos de envelhecimento acelerado e de deterioração controlada para avaliação da qualidade fisiológica de sementes de soja. Scientia Agrícola, Piracicaba, v.52, n.1, p.123-131, 1995.

SANTOS, C.M.R.; MENEZES, N.L.; VILLELA, F.A. Teste de deterioração controlada para avaliação do vigor de sementes de feijão. Revista Brasileira de Sementes, Londrina, v.25, n.2, p.28-35, 2003. 